\title{
Evaluation of disability in patients exposed to fluoroquinolones
}

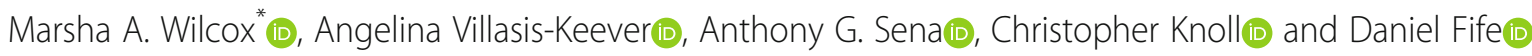

\begin{abstract}
Background: Fluoroquinolones are used for conditions including sinusitis, bronchitis, and urinary tract infections. It has been suggested that exposure to fluoroquinolones for these conditions is associated with disability resulting from adverse events in 2 or more organ systems. The objectives were to: describe: 1) fluoroquinolone, azithromycin, and sulfamethoxazole / trimethoprim utilization for these infections; 2 ) the rate of disability associated with exposure to each of these antibiotic classes and adverse events in 2 or more system organ classes, and 3) compare outcome rates for each of the antibiotic classes.

Methods: This study was conducted using administrative data to mitigate the limitations of spontaneous reports. The sampling frame was a U.S. population with both medical and disability insurance, including patients with the above uncomplicated infections who were prescribed the antibiotics of interest.

The primary outcome was an incident short-term disability claim associated with adverse events in 2 different organ systems within 120 days of exposure. A matched analysis was used to compare the outcome for patients receiving each of the drug classes.

Results: After propensity score matching, there were 119,653 individuals in each of the exposure groups. There were 264 fluoroquinolone associated disability events and 243 azithromycin/ sulfamethoxazole associated disability events (relative risk $=1.09$ (95\% Cl: 0.92-1.30; calibrated $p=0.84)$ ). The results were not significantly different from the null hypothesis of no difference between groups.

Conclusion: Comparative assessments are difficult to conduct in spontaneous reports. This examination of disability associated with adverse events in different system organ classes showed no difference between fluoroquinolones and azithromycin or sulfamethoxazole in administrative data.
\end{abstract}

Keywords: Luoroquinolone, Disability, Azithromycin, Sulfamethoxazole, Administrative data, Adverse events

\section{Key points}

1. It is possible to link disability and administrative claims datasets to evaluate disability as an outcome in a population with both medical and disability insurance.

2. This examination of disability associated with adverse events in more than one system organ class

* Correspondence: mwilcox@its.jnj.com

Janssen Research \& Development, LLC, 1125 Trenton Harbourton Road, Titusville, NJ 08560, USA showed no difference between fluoroquinolones and azithromycin or sulfamethoxazole in administrative data.

\section{Background}

Fluoroquinolones are a broad-spectrum class of antibiotics with high tissue distribution. They are indicated for a wide variety of infections and are among the most frequently prescribed antibiotics. An FDA safety review suggested that the use of fluoroquinolones is associated with disabling and potentially permanent adverse events (AEs) involving 2 or more organ systems that can occur together

(c) The Author(s). 2020 Open Access This article is licensed under a Creative Commons Attribution 4.0 International License, which permits use, sharing, adaptation, distribution and reproduction in any medium or format, as long as you give appropriate credit to the original author(s) and the source, provide a link to the Creative Commons licence, and indicate if changes were made. The images or other third party material in this article are included in the article's Creative Commons licence, unless indicated otherwise in a credit line to the material. If material is not included in the article's Creative Commons licence and your intended use is not permitted by statutory regulation or exceeds the permitted use, you will need to obtain permission directly from the copyright holder. To view a copy of this licence, visit http://creativecommons.org/licenses/by/4.0/. The Creative Commons Public Domain Dedication waiver (http://creativecommons.org/publicdomain/zero/1.0/) applies to the data made available in this article, unless otherwise stated in a credit line to the data. 
in the same patient. The FDA determined that the fluoroquinolones should be reserved for use in patients that have no other options for the following indications: acute sinusitis (AS), acute bacterial exacerbation of chronic obstructive pulmonary disease $(\mathrm{AB})$ and uncomplicated urinary tract infection (UTI). In these indications, the FDA concluded that the risks of these serious side effects generally outweigh the benefits of the use of these antibiotics and all fluoroquinolone labels for systemic use were changed to reflect this recommendation [1].

Once the decision to prescribe an antimicrobial is made, the choice of antimicrobial should be based on an evaluation of both the benefits and adverse events of the antimicrobials available for the specific indication [211].. As with all antimicrobials, the use of fluoroquinolones is limited due to resistance and adverse events [12]. Practice guidelines and reviews by experts consider FQ as alternative to recommended therapy for the treatment of AS [2-4], AB [5-9] and UTI [10, 11].

\section{FDA adverse event reporting system}

The possible association of the use of fluoroquinolones with disabling and potentially permanent adverse events (AEs) was identified from a review of the FDA Adverse Event Report System (FAERS) [1]. The FAERS is a database setup to support the FDA's post-marketing surveillance program by recording adverse events spontaneously reported by consumers and health care professionals to the FDA or manufacturers [13]. This analysis of the spontaneous adverse event reports was conducted without an explicit prior hypothesis and without a comparator. As there is no measure of the total number of patients exposed to a particular drug in a spontaneously reported adverse event database, it is not possible to estimate the rate of adverse events. Janssen, a pharmaceutical company that has marketed a fluoroquinolone, is committed to examining the potential association in a study that would address some of these limitations in administrative claims data.

\section{Objectives}

The primary objectives of this study were to:

Describe drug utilization for fluoroquinolone (FQ), azithromycin (AZ) for sinusitis and bronchitis, and sulfamethoxazole / trimethoprim (ST) for urinary tract infection in an entire health claims database and among those individuals in that database who eligible for short term disability benefits.

Describe the rate of disability associated with 2 or more system organ class adverse events (SOC AEs) among individuals recently exposed FQs or AZ/ST for the indications described above, and
Compare the rates of disability for AEs in 2 or more SOCs after recent exposure to FQs or AZ/ST for these indications (Fig. 1).

\section{Methods \\ Sample}

The sampling frame (the population from which the study patients arose) for this retrospective cohort study was men and women aged 18 through 65 years in a large, well characterized US commercially insured database, IBM MarketScan ${ }^{\circ}$ Commercial database [CCAE] who were eligible for disability insurance and could be linked to the IBM MarketScan ${ }^{\circ}$ Health and Productivity Management database (HPM) during all years for which such data were available, 2007 through 2015. Individuals entered the study on their first exposure to either an FQ or AZ/ST if at the time of that exposure they had been in the database for at least the past 6 months and remained in the database and were insured for disability for at least 120 days afterward, i.e. for the entire time at risk window (see Fig. 1). The date of that exposure was the individual's index date.

\section{IBM MarketScan ${ }^{\oplus}$ Commercial Database (CCAE)}

IBM MarketScan ${ }^{\circ}$ Commercial Database (CCAE) contains data from individuals enrolled in United States employersponsored insurance health plans. The database includes adjudicated health insurance claims (e.g. inpatient, outpatient, and outpatient pharmacy) as well as enrollment data from large employers and health plans who provide private healthcare coverage to employees, their spouses, and dependents. Additionally, it captures laboratory tests for a subset of the covered lives. This administrative claims database includes a variety of fee-for-service, preferred provider organizations, and capitated health plans.

The major data elements contained within this database are outpatient pharmacy dispensing claims (coded with $\mathrm{Na}$ tional Drug Codes (NDC), inpatient and outpatient medical claims which provide procedure codes (coded in Current Procedural Terminology [CPT-4], Healthcare Common Procedure Coding System [HCPCs], International Classification of Diseases, Ninth Revision, Clinical Modification [ICD-9-CM] or ICD-10- Procedure Coding System [PCS]) and diagnosis codes (coded in ICD-9-CM or ICD-10-CM). The data also contain selected laboratory test results (those sent to a contracted thirds-party laboratory service provider) for a non-random sample of the population (coded with Logical Observation Identifiers Names and Codes [LOINC] codes).

\section{IBM MarketScan ${ }^{\circledast}$ Health and Productivity Management Database (HPM)}

IBM MarketScan ${ }^{\oplus}$ Health and Productivity Management Database (HPM) is a subset of the CCAE database, 

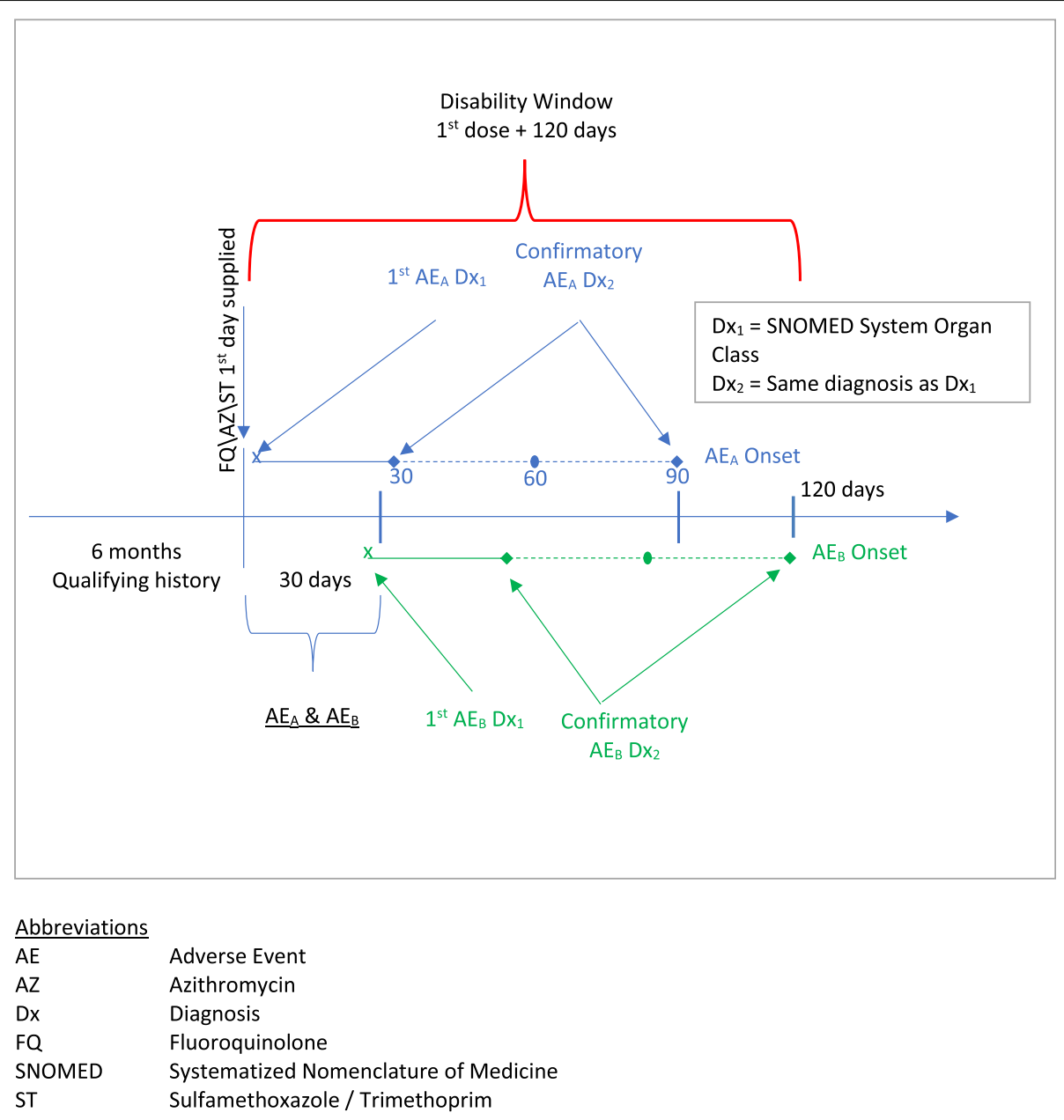

Fig. 1 Study Design

including employees for whom their employer provided information on absences, short-term disability (STD), and workers' compensation. The data in HPM are linkable to the other IBM commercial datasets for these employees.

\section{Indications and exposures}

The sample was limited to individuals who were diagnosed in an outpatient setting with uncomplicated acute bacterial sinusitis, or uncomplicated acute bronchitis and were dispensed an oral fluoroquinolone or azithromycin, but not both; and individuals who had an uncomplicated urinary tract infection (UTI) and were treated with an oral fluoroquinolone or sulfamethoxazole / trimethoprim (e.g. Bactrim), but not both. Exposure was required to occur within 30 days after the indication diagnosis. In case of disagreement between the diagnosis found in the CCAE database and that found in the HPM database, at the recommendation of the data owner, the former was used to identify the indication.
As was done by the FDA, we categorized AEs using the System Organ Class (SOC) in the MedDRA (Medical Dictionary for Regulatory Activities) medical terminology. The SOC is the highest level of term i.e. the level with the broadest terms in the classification. Examples of SOC's include blood and lymphatic system disorders, cardiac disorders, ear and labyrinth disorders, endocrine disorders, eye disorders, musculoskeletal and connective tissue disorders, and psychiatric disorders [14]. The coding algorithms were designed by the authors to approximate the definitions used by the FDA in the analyses of the FAERS data. The algorithms were written into the protocol and the protocol was registered with ClinicalTrials.gov prior to conducting the study. Acute bronchitis was identified using code 466.0 (acute bronchitis). Acute sinusitis was identified using codes 461.0, 461.1, 461.2, 461.3, 461.8, and 461.9, (acute maxillary, frontal, ethmoidal, sphenoidal, other acute sinusitis, acute sinusitis not otherwise specified). Code 599.0 (urinary tract infection, site not specified) was used to identify urinary tract infections. 
The first occurrence of the indication-exposure combination was used for this study. Each study participant qualified only for the first cohort for which he or she was eligible. The samples were mutually exclusive. That is, none of the FQ cohort was exposed to AZ/ST in the prior 6 months. Similarly, none of the AZ/ST cohort was exposed to FQ in the prior 6 months.

\section{General exclusions}

Patients were excluded if they had any of the following conditions, procedures or exposures in the 6 months preceding the first qualifying dose of FQ or AZ/ST: fibromyalgia, rheumatoid arthritis, lupus, diabetes with complications, Lyme disease, multiple sclerosis, renal or hepatic impairment, HIV, joint replacement, or organ transplant; exposure to long-term oral steroid use (30 days or longer) or any cancer chemotherapy, any disability claim.

Condition-specific exclusions were imposed for events within the 3 months preceding the qualifying FQ or AZ/ ST exposure. Patients with acute bronchitis were excluded if they had any of the following: hospitalization for: bronchitis, pneumonia, hypoxemia, respiratory insufficiency; outpatient diagnosis of pneumonia, hypoxemia or respiratory insufficiency. Exclusions for patients with acute sinusitis included: hospitalization for sinusitis or sinus surgery, outpatient sinus surgery or invasive outpatient procedure. Patients with UTI were excluded if they were hospitalized for a UTI, received a catheter or were diagnosed with urinary tract obstruction, pyelonephritis, renal abscess, malformation of the urinary tract, or chronic renal failure.

FQAD was described by FDA as a condition that arises in previously healthy patients after an uncomplicated infection. The exclusions were not for any hospitalization in the past 3 months, but for hospitalizations that were likely to be related to the infections and thus would make it likely that the patient's infection did not qualify as an uncomplicated infection in a previously healthy person. These were not broad exclusions, but were exclusions included to ensure the patients in the study were candidates for the outcome of interest.

\section{Outcomes}

The primary outcome was a disability claim in temporal proximity to confirmed AE's in 2 different MedDRA SOCs among the 6 SOCs of interest (peripheral nervous system, neuropsychiatric, musculoskeletal, sensory, cardiovascular, skin). Disability was defined as an incident short-term disability claim in the HPM database observed within 120 days after the index date (Fig. 1). The disability claim was excluded if it was the continuation of a claim initiated prior to the index date.
Adverse events (AEs) of interest were reported in 2 or more system organ classes. The six categories used in the FDA report were mapped to 7 Medical Dictionary for Regulatory Activities (MedDRA) terms as follows (FDA-MedDRA): cardiovascular- cardiac disorders; sensory-ear and labyrinth disorders; sensory - eye disorders; musculoskeletal- musculoskeletal and connective tissue disorders; peripheral nervous -nervous system disorders; neuropsychiatric - psychiatric disorders; skinskin and subcutaneous tissue disorders.

The IBM CCAE data were mapped to the Observational Medical Outcomes Partnership (OMOP) Common Data Model [15]. As part of this process, the OMOP vocabularies provide a standardized mapping between the ICD-9 codes provided in the IBM CCAE data and their respective related SNOMED standard codes. Additionally, the OMOP vocabulary provides a mapping between SNOMED and the MedDRA System Organ Classes used here. The design for mapping the IBM CCAE data set is maintained at https:/github.com/ OHDSI/ETL-CDMBuilder/tree/master/man/TRUVEN_ CCAE_MDCR_MDCD.

\section{Negative control outcomes}

We chose 45 negative control conditions, conditions believed not to be causally associated with either of the exposure cohorts based on a review of published literature, product labeling and spontaneous adverse event reporting (Supplemental material) to identify residual systematic error in the database or study design, and to empirically calibrate $p$-values for systematic error. For each negative control outcome, we assumed a priori that the true odds ratio (OR) for the outcome was the null value of 1 . We then applied the same analysis used for the study outcomes to each negative control outcome. The difference between the estimated OR for the negative control condition and the expected null value represented an estimate of the systematic error present for that outcome. The distribution of the error estimates from the negative controls was used as the empirical null distribution. We used this distribution to compute a calibrated $p$-value for each outcome $[16,17]$.

\section{Time-at-risk periods}

Our choice of time-at-risk periods was informed by the Briefing Book from the FDA Advisory Panel in 2015 (page 24) [1]:

"The mean and median time to onset of adverse events was 5.4 days and 3 days, respectively. However, the range was very wide, from 1 hour after taking the first dose to 90 days after the drug was discontinued. In almost half of the cases (48\%), the onset was rapid, occurring after one or two doses of 
the drug. In $12 \%$ of the cases, the onset occurred more than 10 days after starting the fluoroquinolone, which in most cases would have been after fluoroquinolone therapy had ended."

If the symptoms are disabling, they should lead to 2 medical encounters within 90 days, and in some cases, much sooner. The appropriate sensitivity analysis, therefore, focused on a shorter, 90-day, at-risk-period.

Qualifying confirmed AEs were incident within 30 days of the first day supplied of the exposure drug with a duration of 30 days or longer. In our primary analysis, in order to be "confirmed" the same diagnosis was required to be observed 30-90 days after the incident diagnosis (Fig. 1). To assess the effect of some of our model assumptions about the length of time from exposure to $\mathrm{AE}$ we conducted a sensitivity analysis in which we shortened the window for the confirmatory diagnosis from 60 days to 30 days.

\section{Comparators}

The purpose of this study was to examine FQAD among patients being treated for uncomplicated acute sinusitis, acute bronchitis, or acute urinary tract infection in an insured population in the U.S. If the occurrence of 2 SOC AEs and disability is associated with FQs, an analogous condition should not also be satisfied for people who received other types of antibiotics, e.g., azithromycin (AZ). Since AZ is not typically used to treat UTI, used Sulfamethoxazole / Trimethoprim (e.g. Bactrim) (ST) as the comparator for that condition.

\section{Statistical analyses}

Crude incidence rates of both outcomes (primary and sensitivity) were estimated within each cohort as the number of individuals with the outcome during each time-at-risk window, divided by the total time-at-risk.

Propensity score adjustment was used as an analytic strategy to reduce potential confounding as the result of imbalance in baseline covariates between the target (FQ) and comparator (AZ/ST) cohorts. The propensity score was the probability of a patient being classified in the target cohort vs. the comparator cohort, given a set of observed covariates. The propensity score was estimated for each patient using the predicted probability from a regularized logistic regression model fit with a Laplace prior (LASSO) and the regularization hyperparameter selected by optimizing the likelihood in a 10-fold cross validation, using a starting variance of 0.01 and a tolerance of 2e-7 [17]. The classes of baseline covariates included in the propensity score model included demographics, diagnoses, drug exposures, and procedures observed in the 30-day, 6-month, and 1-year windows prior to antibiotic exposure [16]. A list of the covariates used in the propensity score can be found in the Supplemental Material.

Propensity score estimates were used to restrict the cohorts through patient trimming. Patients were excluded if their predicted probability was less than $5 \%$ or greater than $95 \%$ of the propensity score distribution across both cohorts. Patients in the target cohort were matched to patients in the comparator cohort using 1:1 matching with a greedy matching algorithm and a caliper of 0.25 of the standard deviation of the propensity score distribution. Standardized mean difference was used as a metric to evaluate the performance of propensity score adjustment.

\section{Comparison}

The outcome model, a conditional Logistic regression, was summarized with the odds ratio and associated 95\% confidence interval. We report effect estimates with nominal $p$-values and empirically calibrated $p$-values [18]. Since the empirical calibration captured systematic error observed from 45 negative controls (Supplemental Material), this statistic was our a priori primary decision criterion for determining statistical significance, including in scenarios where the nominal $p$-value and calibrated $p$-values might have been inconsistent.

\section{Statistical power}

Given matched sample sizes of $119,653, \alpha=0.05$, prevalence of 0.002 , we had $80 \%$ power to detect an odds ratio of 1.25 or greater.

\section{Results}

\section{Sample before matching}

There were more than 10 million $(10,070,296)$ distinct individuals in the CCAE database who were also eligible for disability insurance. Among those, 651,526 individuals were exposed to FQ for any of the qualifying indications; $1,079,158$ were exposed to AZ/ST. The number with full observation time was 204,903 for FQ and 328, 247 for AZ/ST. After study and condition-specific exclusions, there were 141,084 individuals in the FQ and 280, 183 in the AZ/ST unmatched cohorts (Fig. 2).

\section{Sample after matching}

After propensity score matching, there were 119,653 individuals in each of the exposure groups. Figure 2 shows the sample disposition at each step in the sample selection process. Details about the propensity score model can be found in the Supplemental Material. The standardized difference between groups ranged from -.06 (30-34 age group) to 0.23 (dysuria) before matching. After matching, all standardized differences were below 0.1 . The range was -0.03 (female gender) to 0.02 (dysuria) (Table 1). The preference score is a transformation 


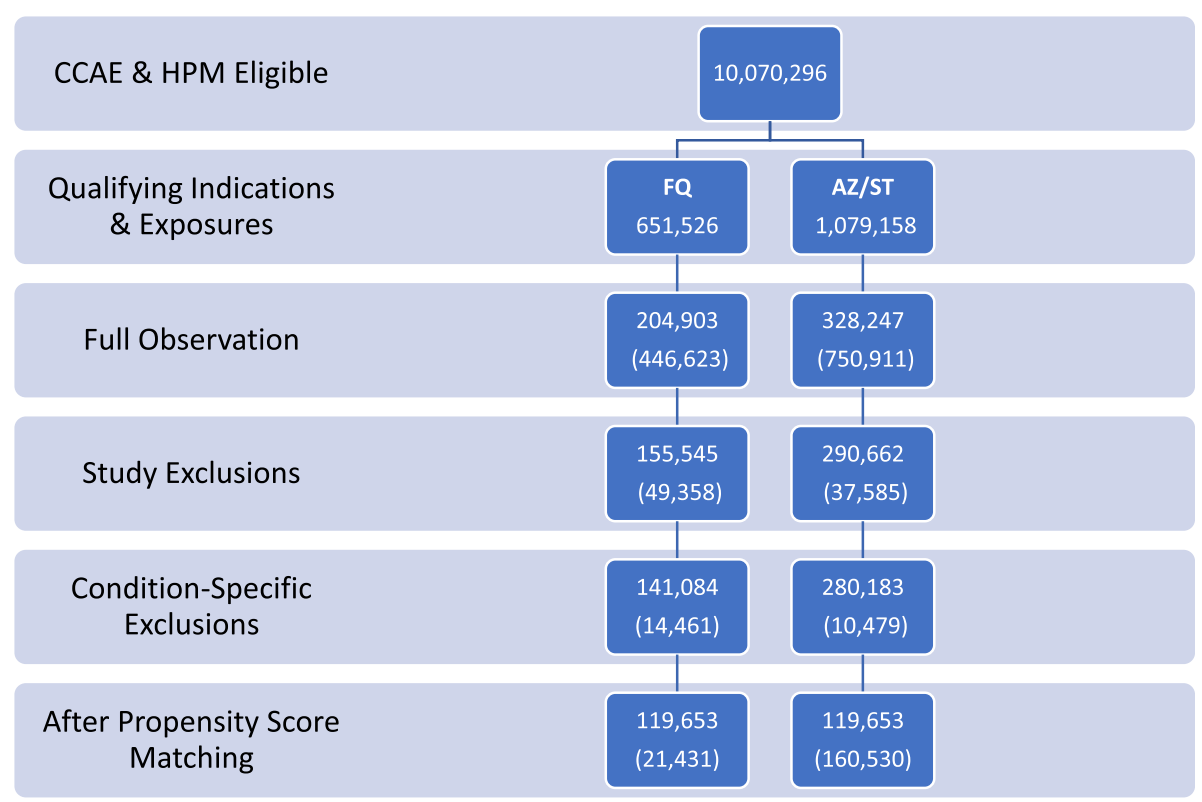

Abbreviations: $\mathrm{CCAE}=$ Truven MarketScan Commercial Claims and Encounters database; HPM= MarketScan ${ }^{\circledR}$ Health and Productivity Management database; $A Z$ =azithromycin; $F Q=$ fluoroquinolone; $S T=$ sulfamethoxazole / trimethoprim; Numbers in parentheses () are counts of patients excluded at each step

Fig. 2 Sample Disposition

of the propensity score that adjusts for differences in the sizes of the two treatment groups. The preference score plot shows the distribution of the score in each of the samples before and after matching (Fig. 3). Overlap of the distributions indicates subjects in the two groups were similar in terms of their predicted probability of receiving one treatment over the other.

\section{Antibiotic use for indications of interest after matching}

Azithromycin was used more than any of the fluoroquinolones to treat sinusitis $(59,501$ vs. 48,170$)$. Among the fluoroquinolones, levofloxacin was most often used for this indication. Similarly, azithromycin was used more often to treat bronchitis $(47,933$ vs. 31,503$)$, with levofloxacin the most often used fluoroquinolone. For UTI, fluoroquinolones were prescribed more often than sulfamethoxazole / trimethoprim, (57,676 vs. 22,700). Ciprofloxacin was used ten times more often than the next most often used FQ, levofloxacin (Table 2). Ciprofloxacin and levofloxacin accounted for the majority of fluoroquinolone use overall. Levofloxacin was used most for the treatment of sinusitis and bronchitis, while ciprofloxacin was used most for urinary tract infections.

\section{Antibiotic-associated disability (FQ AD/AZST AD)}

There were 264 cases of FQAD. Among those, 117 were exposed to levofloxacin, 111 to ciprofloxacin, 34 to moxifloxacin, and 2 to Gemifloxacin (Table 3). There were 243 cases of antibiotic associated disability among those exposed to azithromycin or sulfamethoxazole / trimethoprim.

Descriptive statistics about the cases in both cohorts can be found in Table 4. The median age of cases in the FQ cohort was 49; 51 in the AZ/ST cohort. Women comprised $55.3 \%$ of the FQ cohort; $62.6 \%$ of the AZ/ST group. In the non-elderly population, UTI's are more common in women than in men, and ST is very often used to treat uncomplicated UTI's.

Roughly $1 / 3$ of each group were treated for sinusitis. In the FQ cohort, $17 \%$ were treated for bronchitis; the number was nearly twice that (37\%) for the AZ/ST group. Close to $1 / 3$ of the FQ group were treated for cystitis or a UTI; only $13 \%$ in the AZ/ST group were treated for this indication. Among those exposed to FQ, $55.3 \%$ were women, the same was true for $62.6 \%$ of the AZ/ST cohort. The median time to AE onset was 8 days for both groups. The median time to confirmatory diagnosis was 42 days for the FQ group; 44 days in the AZ/ ST group. The range for both groups was 30-90 days.

\section{Comparison of rates of FQ AD with AZ/ST AD}

There were 264 FQAD events and 243 AZ/ST events in the matched samples (Table 5). The observed crude odds ratio was 1.09 (95\% CI: 0.92-1.30). The $p$-value for the adjusted odds ratio was $p=0.35$; the calibrated $p$ value was $p=0.84$. Calibration results can be found in the Supplemental Material. The results were not significantly different from the null hypothesis of no difference between groups. 
Table 1 Sample characteristics before and after matching

\begin{tabular}{|c|c|c|c|c|c|c|}
\hline \multirow{4}{*}{$\begin{array}{l}\text { Characteristic } \\
\mathrm{N}\end{array}$} & \multicolumn{3}{|c|}{ Before matching } & \multicolumn{3}{|c|}{ After matching } \\
\hline & \multirow{2}{*}{$\begin{array}{l}\mathrm{FQ} \\
155 \\
776\end{array}$} & \multicolumn{2}{|c|}{$\mathrm{AZZST}$} & \multirow{2}{*}{$\begin{array}{l}\mathrm{FQ} \\
119 \\
653\end{array}$} & \multicolumn{2}{|c|}{$\mathrm{AZ} / \mathrm{ST}$} \\
\hline & & $\begin{array}{l}294, \\
663\end{array}$ & & & $\begin{array}{l}119 \\
653\end{array}$ & \\
\hline & \multicolumn{2}{|c|}{ Percent } & $\begin{array}{l}\text { Standardized } \\
\text { Difference }\end{array}$ & \multicolumn{2}{|c|}{ Percent } & $\begin{array}{l}\text { Standardized } \\
\text { Difference }\end{array}$ \\
\hline \multicolumn{7}{|l|}{ Age group } \\
\hline $15-19$ & 0 & 0 & 0 & 0 & 0 & 0 \\
\hline $20-24$ & 0.9 & 1 & -0.01 & 0.9 & 0.9 & 0 \\
\hline $25-29$ & 5.4 & 6.3 & -0.04 & 5.7 & 5.5 & 0.01 \\
\hline $30-34$ & 9 & 10.8 & -0.06 & 9.4 & 9.1 & 0.01 \\
\hline $35-39$ & 12.3 & 14 & -0.05 & 12.6 & 12.3 & 0.01 \\
\hline $40-44$ & 15.4 & 16.1 & -0.02 & 15.5 & 15.2 & 0.01 \\
\hline $45-49$ & 17.5 & 17 & 0.01 & 17.4 & 17.5 & 0 \\
\hline $50-54$ & 18.3 & 17 & 0.04 & 18.1 & 18.4 & -0.01 \\
\hline $55-59$ & 14.9 & 12.8 & 0.06 & 14.4 & 14.9 & -0.01 \\
\hline $60-64$ & 6.2 & 4.9 & 0.06 & 5.9 & 6.2 & -0.01 \\
\hline $65-69$ & 0.1 & 0 & 0.01 & 0.1 & 0.1 & 0 \\
\hline Female gender & 59.1 & 49.3 & 0.2 & 57 & 58.6 & -0.03 \\
\hline \multicolumn{7}{|l|}{ Medical history } \\
\hline Dysuria & 6 & 1.7 & 0.23 & 3.9 & 3.5 & 0.02 \\
\hline Hematuria syndrome & 3.2 & 0.9 & 0.16 & 1.9 & 1.7 & 0.01 \\
\hline \multicolumn{7}{|l|}{ Medication use } \\
\hline Phenazopyridine hydrochloride $200 \mathrm{mg}$ oral tablet & 5.3 & 1.3 & 0.23 & 3.1 & 2.8 & 0.02 \\
\hline $\begin{array}{l}\text { Nitrofurantoin, Macrocrystals } 25 \mathrm{mg} \text { / Nitrofurantoin, Monohydrate } \\
75 \mathrm{mg} \text { oral capsule }\end{array}$ & 9.9 & 5.6 & 0.16 & 7.9 & 7.9 & 0 \\
\hline Amoxicillin $875 \mathrm{mg}$ / Clavulanate $125 \mathrm{mg}$ oral tablet & 9.2 & 4.9 & 0.17 & 7.6 & 8.1 & -0.02 \\
\hline
\end{tabular}

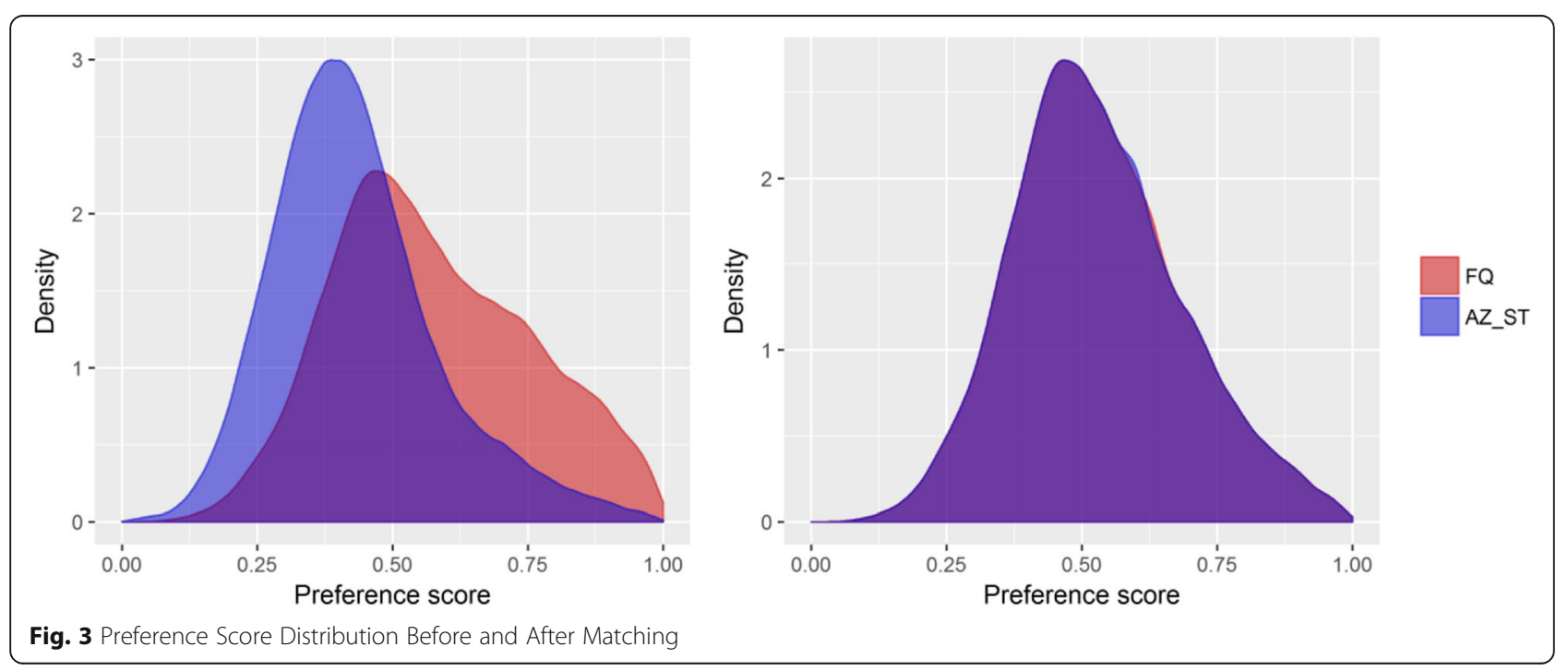


Table 2 Indications of interest treated by antibiotics of interest after matching

\begin{tabular}{lllll}
\hline & Sinusitis & Bronchitis & $\mathrm{UTI}^{\mathrm{a}}$ & Any the Conditions \\
\hline Levofloxacin & 31,065 & 21,723 & 5170 & 49,923 \\
Ciprofloxacin & 8535 & 4226 & 52,307 & 56,792 \\
Moxifloxacin & 8178 & 5143 & 175 & 12,117 \\
Ofloxacin & 7 & 4 & 45 & 52 \\
Gemifloxacin & 420 & 424 & 13 & 785 \\
Gatifloxacin & 1 & 0 & 1 & 2 \\
Norfloxacin & 0 & 1 & 4 & 5 \\
FQ Total & 48,170 & 31,503 & 57,676 & 119,653 \\
AZ/ST & 59,501 & 47,933 & 22,700 & 119,653 \\
AZ & 59,206 & 47,886 & 461 & 99,871 \\
ST & 328 & 75 & 22,255 & 19,782 \\
\hline
\end{tabular}

Abbreviations: AZ Azithromycin, FQ Fluoroquinolone, ST Sulfamethoxazole / Trimethoprim, UTI Urinary tract infection

${ }^{\mathrm{a} U T I}$ indication was treated by ST

${ }^{\mathrm{b}} \mathrm{AZ}$ exposure required indication of sinusitis/bronchitis and ST required UTI. Other indications may co-occur with these treatments

Table 6 shows the distribution of SOC AEs in the cases. Cases in the FQ group had an average of 2.66 AEs. The average was 2.64 in the AZ/ST cohort.

\section{Sensitivity analysis}

In the sensitivity analysis, restricting the observation window to 30 days, there were 205 events in the FQ cohort and 182 events in the AZ/ST cohort with an adjusted odds ratio of 1.13. The $p$-value for the adjusted odds ratio was $p=0.24$; the calibrated $p$-value was $p=0.89$. The results for the sensitivity analysis were not different from the hypothesis of no difference between groups.

While the counts were lower, the inference was the same; no difference between groups in the incidence of antibiotic-associated disability (Table 5). Detailed results from the sensitivity analyses can be found in the Supplemental Material.

We examined the distribution of time to the second, confirmatory, diagnosis in both our primary 120-day window and the 90-day window in the sensitivity analysis. The median time to confirmatory diagnosis was 42/44 days (FQAD/AZSTAD) in the primary 120-day analysis and 37/40 days in the 90-day sensitivity analysis.

\section{Limitations}

There were several limitations in this work. The source population was limited to administrative healthcare claims among a privately insured population with disability insurance. Our definition of disability required employment and therefore excluded the elderly and the unemployed populations. The disability data were not perfectly matched to the medical claims because not all the CCAE database contributors were able to supply all types of HPM data for every data year.

The average dwell time in such databases is approximately 2 years. Qualifying events that began prior to the insurance coverage or persisted afterward were censored. Similarly, events that began prior to the observation period and exposures that occurred prior to the observation period were missed.

We necessarily made assumptions about the allowable time between the first and second diagnosis and the allowable time for filing a claim. Though we did sensitivity analyses, it remains possible that different choices of these times would have yielded different estimates of the relative risk. We conducted a sensitivity analysis about the time to qualifying adverse events. The point estimate was similar and the inference was the same.

There are several ways in which our study design could introduce bias. First, we require patients to have 120 days of observation post exposure. Patients for

Table 3 AE count and disability for each fluoroquinolone (counts) ${ }^{a}$

\begin{tabular}{|c|c|c|c|c|c|}
\hline & \multirow[b]{2}{*}{$\begin{array}{l}\text { Count Used for Any } \\
\text { Condition }\end{array}$} & $\begin{array}{l}1 \text { confirmed qualifying } \\
A E\end{array}$ & $\begin{array}{l}1 \text { confirmed qualifying } \\
\mathrm{AE} \\
+ \text { Disability }\end{array}$ & $\begin{array}{l}2+\text { confirmed qualifying } \\
\text { AEs }\end{array}$ & $\begin{array}{l}2+\text { confirmed qualifying } \\
\text { AEs } \\
+ \text { Disability }\end{array}$ \\
\hline & & \multicolumn{4}{|l|}{ Count (Row \%) } \\
\hline Levofloxacin & 49,923 & 7559 (15.1\%) & $472(0.9 \%)$ & $1328(2.7 \%)$ & $117(0.2 \%)$ \\
\hline Ciprofloxacin & 56,792 & 7062 (12.4) & $530(0.9)$ & $1109(2.0)$ & $111(0.2)$ \\
\hline Moxifloxacin & 12,117 & $1863(15.4)$ & $105(0.9)$ & $281(2.3)$ & $34(0.3)$ \\
\hline Ofloxacin & 52 & $6(11.5)$ & $0(0.0)$ & $1(1.9)$ & $0(0.0)$ \\
\hline Gemifloxacin & 785 & $110(14.0)$ & $5(0.6)$ & $16(2.0)$ & $2(0.3)$ \\
\hline Gatifloxacin & 2 & 0 & $0(0.0)$ & $0(0.0)$ & $0(0.0)$ \\
\hline Norfloxacin & 5 & 0 & $0(0.0)$ & $0(0.0)$ & $0(0.0)$ \\
\hline TOTAL & 119,676 & 16,600 & 1112 & 2735 & 264 \\
\hline
\end{tabular}

Abbreviations: $A E$ Adverse event

${ }^{a}$ Counts above are not mutually exclusive 
Table 4 Descriptive statistics for antibiotic associated disability cases

\begin{tabular}{|c|c|c|c|c|c|}
\hline & & $\mathrm{FQ} \mathrm{AD}$ & & $\mathrm{AZ/ST} A D$ & \\
\hline & & $N=264$ & & $N=243$ & \\
\hline & & Count & $\%$ & Count & $\%$ \\
\hline Age & Mean (sd) & $48.5(8.2)$ & & $48.8(8.7)$ & \\
\hline & Median & 49 & & 51 & \\
\hline & Range & $26-64$ & & $24-64$ & \\
\hline & 18-29years & 5 & 1.9 & 6 & 2.5 \\
\hline & $30-59$ years & 238 & 90.2 & 218 & 89.7 \\
\hline & $>=60$ years & 21 & 8.0 & 19 & 7.8 \\
\hline Gender & Female & 146 & 55.3 & 152 & 62.6 \\
\hline & Male & 118 & 44.7 & 91 & 37.4 \\
\hline Non-UTI Cases ${ }^{a}$ & Total & 164 & 62.1 & 207 & 85.2 \\
\hline & Female & 73 & 44.5 & 122 & 58.9 \\
\hline & Male & 91 & 55.5 & 85 & 41.1 \\
\hline Indication & Sinusitis & 97 & 33.1 & 96 & 35.3 \\
\hline & Bronchitis & 50 & 17.1 & 100 & 36.8 \\
\hline & Cystitis/UTI & 100 & 34.1 & 36 & 13.2 \\
\hline & Sinusitis/bronchitis & 15 & 5.1 & 9 & 3.3 \\
\hline & Bronchitis/UTI & 2 & 0.7 & 2 & 0.7 \\
\hline Days to AE Onset & Mean (sd) & $9.7(7.1)$ & & $10.5(7.8)$ & \\
\hline & Median & 8 & & 8 & \\
\hline & Range & $1-30$ & & $1-29$ & \\
\hline & $1-2$ days & 41 & 15.5 & 38 & 15.6 \\
\hline & $3-4$ days & 33 & 12.5 & 29 & 11.9 \\
\hline & $5-10$ days & 88 & 33.3 & 77 & 31.7 \\
\hline & $>10$ days & 102 & 38.6 & 99 & 40.7 \\
\hline Days to Confirmatory Diagnosis & Mean (sd) & $46.7(16.0)$ & & $49.0(16.0)$ & \\
\hline Range $30-90$ days & Median & 42 & & 44 & \\
\hline
\end{tabular}

Abbreviations: AZ Azithromycin, FQ Fluoroquinolone, ST Sulfamethoxazole / Trimethoprim, UTI Urinary tract infection

${ }^{\text {a } T h e ~ c o u n t ~ o f ~ N o n-U T I ~ c a s e s ~ i n c l u d e s ~ i n d i c a t i o n s ~ f o r ~ b r o n c h i t i s ~ a n d ~ s i n u s i t i s . ~ W h e n ~ t h e s e ~ i n d i c a t i o n s ~ o c c u r r e d ~ i n ~ c o m b i n a t i o n ~ w i t h ~ U T I, ~ t h e y ~ w e r e ~ i n c l u d e d ~ i n ~}$ this count

Table 5 Outcome - crude and adjusted odds ratios (ORs)

\begin{tabular}{|c|c|c|c|c|c|c|}
\hline & $\begin{array}{l}\mathrm{FQ} \\
(\mathrm{N}, \text { col. \%) }\end{array}$ & $\begin{array}{l}\text { AZ/ST } \\
(\mathrm{N}, \text { col. \%) }\end{array}$ & $\begin{array}{l}\mathrm{FQ} \\
(95 \% \mathrm{Cl})\end{array}$ & $\begin{array}{l}\text { AZ/ST } \\
(95 \% \mathrm{Cl})\end{array}$ & $\begin{array}{l}\text { Crude OR } \\
(95 \% \mathrm{Cl})\end{array}$ & $\begin{array}{l}\text { Adjusted OR } \\
(95 \% \mathrm{Cl})\end{array}$ \\
\hline $\mathrm{N}$ & 119,653 & 119,653 & & & & \\
\hline \multirow{4}{*}{$\begin{array}{l}>=2 \text { SOC AEs } \\
+ \text { Disability }\end{array}$} & 264 & 243 & 0.002 & 0.002 & 1.09 & 1.09 \\
\hline & $0.22 \%$ & $0.20 \%$ & $(0.002-0.002)$ & $(0.002-0.002)$ & $(0.92-1.30)$ & $(0.91-1.30)$ \\
\hline & & & & & & $p=0.35$ \\
\hline & & & & & & calibrated $p=0.84$ \\
\hline \multicolumn{7}{|c|}{ Sensitivity Analyses } \\
\hline \multirow{4}{*}{$\begin{array}{l}>=2 \text { SOC AEs } \\
+ \text { Disability }\end{array}$} & 205 & 182 & 0.002 & 0.002 & 1.13 & 1.13 \\
\hline & $0.17 \%$ & $0.15 \%$ & $(0.001-0.002)$ & $(0.001-0.002)$ & $(0.92-1.37)$ & $(0.92-1.38)$ \\
\hline & & & & & & $p=0.24$ \\
\hline & & & & & & calibrated $p=0.89$ \\
\hline
\end{tabular}


Table 6 System Organ Class AEs in the Cases

\begin{tabular}{lll}
\hline \multirow{2}{*}{$\begin{array}{ll}\text { System Organ } \\
\text { Class }\end{array}$} & FQ AD & AZ/ST AD \\
\cline { 2 - 3 } Peripheral nervous & $\boldsymbol{n}=264$ & $\boldsymbol{n}=243$ \\
Neuropsychiatric & $131(0.11 \%)$ & $87(0.07 \%)$ \\
Musculoskeletal & $103(0.09 \%)$ & $87(0.07 \%)$ \\
Sensory & $75(0.06 \%)$ & $139(0.12 \%)$ \\
Cardiovascular & $161(0.13 \%)$ & $197(0.16 \%)$ \\
Skin & $209(0.17 \%)$ & $115(0.10 \%)$ \\
Total & $24(0.02 \%)$ & $16(0.01 \%)$ \\
Average per case & 703 & 641 \\
\hline
\end{tabular}

whom the AEs were severe may have died or lost their insurance and would be lost to the study. In our effort to replicate the FDA study, we required 2 AEs prior to the disability claim. Patients with mortality related to a single AE would not have the opportunity to be counted in our work. Further, we required a confirmation of each of the AEs during a 30-day window, thereby introducing immortal time bias. This too, has the potential for biasing our findings."

Our findings should be interpreted in light of the limitations inherent in claims-based analyses. The results of this work may not be generalizable to populations not included in the study (e.g., patients who are uninsured).

\section{Discussion}

The benefit-risk profile of antibiotics is relatively easy to discern when the infection is severe and the burden of disease great. It can be a more challenging question when the infection is less severe and is uncomplicated. It was in this context the idea of fluoroquinolone-associated disability arose in spontaneous report data. While these data include a reporter, an outcome and a drug, the reports do not have a known denominator with which to estimate and compare rates. We sought to evaluate the characteristics of FQAD, including the question of whether it is unique to FQ's or whether a similar pattern of adverse events and disability occurs with the use of other antibiotics used to treat the same conditions. We conducted the work in a large US administrative claims database in which the denominator would be known. To that end, we compared the disability rate in fluoroquinolones with the rates observed with the use of AZ/ST, when prescribed for the indications of interest.

Current FDA labeling for fluoroquinolones carries a Boxed Warning that appears to be based on FQAD in that it speaks of disabling and potentially irreversible serious adverse reactions that have occurred together, names several body systems that may be affected, and, for each fluoroquinolone, states that for treatment of uncomplicated urinary tract infection, acute bacterial exacerbation of chronic bronchitis, or acute bacterial sinusitis the use of fluoroquinolone should be reserved for patients who have no alternative options. FDA announcements such as the one at https://www.fda.gov/ drugs/information-drug-class/fda-approves-safety-labeling-changes-fluoroquinolones, also suggest that this language is essentially warning about FQAD.

The present study offers evidence that such serious disabling and potentially irreversible adverse reactions that have occurred together are infrequent (incidence of $0.2 \%$ ) and not unique to fluoroquinolones but also occur at approximately the same frequency after exposure to azithromycin sulfamethoxazole / trimethoprim for the same three indications among new users in the first 30 days after the start of exposure.

Our findings have important implications for understanding the safety profile of antimicrobials. When a decision to prescribe antimicrobials is made, the choice of antimicrobial should be based on an evaluation of both the potential benefits and adverse events of the antimicrobials available for the specific indication. For some indications, the benefit of the use of antimicrobials is limited: in acute sinusitis where the prevalence of bacterial infection is only $2-10 \%$, and up to $80 \%$ of cases improve spontaneously; in mild cases of acute bacterial exacerbation of chronic bronchitis the effect of antibacterial drugs is modest, and its routine use is therefore not recommended. In these cases, the use of any antimicrobial should be limited to those cases where there is clear evidence of potential benefit.

\section{Conclusion}

In propensity-score matched sample from a defined US working population with disability insurance, we found no difference between the incidence of disability associated with AE's in two SOCs between those exposed to FQ's and those exposed to AZ/ST.

\section{Supplementary information}

Supplementary information accompanies this paper at https://doi.org/10. 1186/s40360-020-00415-4.

\footnotetext{
Additional file 1: Fluoroquinolone and Disability - Negative Control Outcomes and Propensity Score Model. The file contains a list of the negative control outcomes used and the resultant $p$-value calibration. The file also contains a description of covariates evaluated for inclusion in the propensity score model and a reference to the accompanying excel file containing model parameters.

Additional file 2:. Model parameters for propensity scores. Model details for propensity scores.
}

\section{Abbreviations}

ABECB: Acute bacterial exacerbation of chronic obstructive pulmonary disease; AE: Adverse Event; AS: Acute sinusitis; AZ: Azithromycin; CCAE: IBM MarketScan ${ }^{\oplus}$ Commercial database; CPT: Current Procedural Terminology; Dx: Diagnosis; FAERS: FDA Adverse Event Reporting System; 
NFQ: Fluoroquinolone; HCPCS: Healthcare Common Procedure Coding System; HPM: Health and Productivity Management Database; ICD-9CM: International Classification of Diseases, 9th Revision, Clinical Modification; ICD-10-PCS: International Classification of Disease -10th Revisions, Procedure Coding System; LOINC: Logical Observation Identifiers Names and Codes; OMOP: Observational Medical Outcomes Partnership; NDC: National Drug Codes; SNOMED: Systematized Nomenclature of Medicine; SOC: System organ class; ST: Sulfamethoxazole / Trimethoprim; UTI: Urinary tract infection

\section{Acknowledgements}

The authors would like to acknowledge Patrick Ryan for methods advice and Sergio Fonseca for clinical consultation.

\section{Authors' contributions}

All authors approved the submitted version of the manuscript and have agreed to be personally for their contributions. MW: Made contributions to the conception, design of the work, interpretation of the data, and drafted the manuscript. AVK: Made contributions to the design of the work, interpretation of the data, and drafting of the manuscript. AS: Made contributions to the design of the work, acquired the data, conducted the analysis, and drafting of the manuscript. CK: Made contributions to the design of the work, acquired the data, conducted the analysis, and reviewed the manuscript. DF: Made contributions to the conception, design of the work, interpretation of the data, and edited the manuscript.

\section{Funding}

This work was funded by Janssen Pharmaceutical R\&D, LLC., a Johnson \& Johnson company. The authors were responsible for design of the study, analysis, and interpretation of the data as well as writing the manuscript. Janssen had a license for the IBM MarketScan ${ }^{\oplus}$ Commercial database.

\section{Availability of data and materials}

The data that support the findings of this study are available from IBM MarketScan ${ }^{\oplus}$ but restrictions apply to the availability of these data, which were used under license for the current study, and so are not publicly available. Data are however available from the authors upon reasonable request and with permission of IBM MarketScan ${ }^{\oplus}$

\section{Ethics approval and consent to participate}

The use of IBM MarketScan ${ }^{\oplus}$ Commercial Database was reviewed by the New England Institutional Review Board (IRB) and was determined to be exempt from broad IRB approval, as this research project did not involve human subjects research.

\section{Consent for publication}

Not applicable.

\section{Competing interests}

All authors are employees of Janssen Pharmaceuticals, LLC, a Johnson \& Johnson company, and shareholders in Johnson \& Johnson. Janssen markets a fluoroquinolone in some countries.

Received: 5 April 2019 Accepted: 19 May 2020

Published online: 03 June 2020

\section{References}

1. FDA Drug Safety Communication: FDA updates warnings for oral and injectable fluoroquinolone antibiotics due to disabling side effects (2016). Available from: https://www.fda.gov/downloads/Drugs/DrugSafety/UCM513 019.pdf.

2. Ahovuo-Saloranta A, Rautakorpi UM, et al. Antibiotics for acute maxillary sinusitis in adults. Cochrane Database Syst Rev. 2014;2014(Issue 2): CD000243. https://doi.org/10.1002/14651858.CD000243.pub3.

3. Young J, De Sutter A, Merenstein D, et al. Antibiotics for adults with clinically diagnosed acute Rhinosinusitis: a meta-analysis of individual patient data. Lancet. 2008:371:908-14.

4. Chow AW. Benninger MS, et al; Infectious Diseases Society of America. Clin Infect Dis. 2012;54(8):e72-e112. https://doi.org/10.1093/cid/cir1043 Epub 2012 Mar 20.
5. Llor C, Bjerrum L. Antibiotic prescribing for acute bronchitis. Expert Rev Anti-Infect Ther. 2016;14(7):633-42. https://doi.org/10.1080/14787210.2016. 1193435.

6. Harris AM, Hicks LA, Qaseem A. Appropriate antibiotic use for acute respiratory tract infection in adults: advice for high-value care from the American College of Physicians and the Centers for Disease Control and Prevention. Ann Intern Med. 2016;164:425-34.

7. Bach PB, Brown C, Gelfand SE, et al. Management of Acute Exacerbations of chronic obstructive pulmonary disease: a summary and appraisal of published evidence. Ann Intern Med. 2001;134:600-20.

8. Sethi S, Murphy TF. Infection in the pathogenesis and course of chronic obstructive pulmonary disease. New Engl J Med. 2008;359:2355-65.

9. Celli BR, MacNee W, et al. Standards for the diagnosis and treatment of patients with COPD: a summary of the ATS/ERS position paper. Eur Respir J. 2004;23:932-46

10. Zalmanovici Trestioreanu A, Green H, Paul M, Yaphe J, Leibovici L. Antimicrobial agents for treating uncomplicated urinary tract infection in women. Cochrane Database Syst Rev. 2010;2010(Issue 10):CD007182. https:// doi.org/10.1002/14651858.CD007182.pub2.

11. Gupta $\mathrm{K}$, Hooton $\mathrm{TM}$, Naber $\mathrm{KG}$, et al. International clinical practice guidelines for the treatment of acute uncomplicated cystitis and pyelonephritis in women: a 2010 update by the Infectious Diseases Society of America and the European Society for Microbiology and Infectious Diseases. Clin Infect Dis. 2011:52(5):e103-20.

12. Tandan M, Cormican M, Vellinga A. Adverse events of fluoroquinolones vs otherantimicrobials prescribed in primary care: a systematic review and meta-analysis of randomized controlled trials. Int J Antimicrob Agents. 2018; 52(5):529-40.

13. FDA Adverse Event Reporting System (FAERS) 2012. Available from: http:// www.fda.gov/Drugs/GuidanceComplianceRegulatoryInformation/ Surveillance/AdverseDrugEffects/default.htm.

14. Introductory Guide, MedDRA Version 21.0, March 2018: https://www. meddra.org/sites/default/files/guidance/file/intguide_21_0_english.pdf. Accessed 24 July 2019.).

15. Voss, et al. Feasibility and utility of applications of the common data model to multiple, disparate observational health databases. J Am Med Inform Assoc. 2015:22(3):553-64. https://doi.org/10.1093/jamia/ocu023.

16. Ryan PB, Schuemie MJ, Welebob E, Duke J, Valentine S, Hartzema AG. Defining a reference set to support methodological research in drug safety. Drug Saf. 2013;36(suppl 1):S33-47 [PubMed]

17. Tian Y, Schuemie MJ, Suchard MA. Evaluating large-scale propensity score performance through real-world and synthetic data experiments. Int J Epi. 2018;47(6):2005-14. https://doi.org/10.1093/ije/dyy120.

18. Schuemie MJ, Ryan PB, DuMouchel W, Suchard MA, Madigan D. Interpreting observational studies: why empirical calibration is needed to correct $p$ values. Stat Med. 2014;33:209-18 [PubMed].

\section{Publisher's Note}

Springer Nature remains neutral with regard to jurisdictional claims in published maps and institutional affiliations.

Ready to submit your research? Choose BMC and benefit from:

- fast, convenient online submission

- thorough peer review by experienced researchers in your field

- rapid publication on acceptance

- support for research data, including large and complex data types

- gold Open Access which fosters wider collaboration and increased citations

- maximum visibility for your research: over $100 \mathrm{M}$ website views per year

At BMC, research is always in progress.

Learn more biomedcentral.com/submission 